\title{
Efficacy and Safety of the Losartan-Hydrochlorothiazide Combination Tablet in Patients with Hypertension Uncontrolled by Angiotensin II Receptor Antagonist Therapy: The Aichi Research on Combination Therapy for Hypertension (ARCH) Study
}

\author{
Kengo Maeda ${ }^{1}$, Masayoshi Adachi ${ }^{2}$, Atsushi Kinoshita ${ }^{3}$, Naoki Koh ${ }^{4}$, \\ Yoshitaka Miura $^{5}$ and Toyoaki Murohara ${ }^{1}$
}

\begin{abstract}
Background The guidelines recommend combination therapy for patients who are unable to achieve target BP with monotherapy; some fixed dose therapies including an angiotensin II receptor blocker (ARB) and diuretics are available in Japan. However, to date there have been few reports on this long-term treatment and the patient profiles suited for this combination remain ambiguous.

Method The Aichi Research on Combination therapy for Hypertension Study was a multicenter, open-label, prospective observational study that investigated the efficacy and safety of 1-year treatment with the losartanhydrochlorothiazide (HCTZ) combination tablet in patients with hypertension uncontrolled by either ARB monotherapy or combination therapy with a calcium channel blocker (CCB). An ARB was switched to a losartan-HCTZ tablet after a pre-observation period.

Results A total of 614 of 648 patients were evaluable (mean age, 66.3 years; $52.8 \%$ men; mean baseline blood pressure, BP, $157.7 / 87.9 \mathrm{mmHg}$ ). The BP had decreased significantly to $138.0 / 78.2 \mathrm{mmHg}$ by month 3 $(\mathrm{p}<0.001, t$-test), and $36.2 \%$ of the patients had achieved their target BP. The hypotensive effect lasted for 1 year and was found equally in the losartan-HCTZ arm and the losartan-HCTZ plus CCB arm. A stratified analysis showed significant hypotensive effects in patients with higher baseline BP, women, and patients who did not drink alcohol ( $<<0.001$, unpaired $t$-test).

Conclusion The losartan-HCTZ combination tablet was found to have an early hypotensive effect, good tolerability, and stable long-term benefits in patients with hypertension uncontrolled by ARB monotherapy or combination therapy with a CCB.
\end{abstract}

Key words: angiotensin II receptor antagonist, antihypertensive agent, hydrochlorothiazide, hypertension, losartan

(Intern Med 51: 1167-1175, 2012)

(DOI: 10.2169/internalmedicine.51.6297)

\section{Introduction}

There has been tremendous progress in the development of antihypertensive agents, consequently it is now much easier to control blood pressure (BP) in patients with hyperten- sion. Nevertheless, many patients fail to achieve BP control. The international guidelines, including those of the Japanese Society of Hypertension (JSH), recommend combination therapy for patients unable to achieve their target BP with monotherapy. One such combination therapy is an angiotensin II receptor antagonist (angiotensin receptor

${ }^{1}$ Department of Cardiology, Nagoya University Graduate School of Medicine, Japan, ${ }^{2}$ Adachi Clinic Internal Medicine and Cardiovascular Medicine, Japan, ${ }^{3}$ Kinoshita Clinic, Japan, ${ }^{4}$ Koh Internal Medicine Clinic, Japan and ${ }^{5}$ Miura Internal Medicine Clinic, Japan Received for publication August 1, 2011; Accepted for publication December 26, 2011 Correspondence to Dr. Kengo Maeda, maedaken@med.nagoya-u.ac.jp 
blocker, ARB) and a diuretic agent. The combination of the traditional high intake of sodium and the recent increase in the number of individuals with metabolic syndrome has emerged as an important problem of public health in Japan. Excessive sodium intake in particular is a major cause of hypertension in Japan. It is generally accepted that reducing sodium intake could reduce BP (1), but sufficient dietary sodium restriction is not achievable in most elderly in Japan.

Diuretic agents block the reabsorption of sodium by uriniferous tubules, and thereby reduce body fluid volume and cardiac output. These effects, in turn, shift the pressurenatriuresis-diuresis curve of patients with salt-sensitive hypertension toward that of patients with non-salt-sensitive hypertension. Therefore, diuretic agents seem to be particularly effective in reducing BP in salt-sensitive patients, in patients such as those who ingest excessive amounts of salt, patients with diabetes mellitus, women, and patients with chronic kidney disease. The combination of antihypertensive agents in a single tablet would be expected to improve dose compliance, which would thus facilitate hypertensive patients to achieve better control of their BP (2). Therefore, some fixed dose medications that include an ARB and a diuretic agent have been approved and are clinically used in Japan. This combination has a synergistic effect and is expected to reduce BP more effectively. However, to date there have been few reports on the long-term treatment, and the patient profiles that are better suited for this combination treatment remain to be elucidated.

Therefore, this study investigated the efficacy and safety of intensive treatment in which an ARB was replaced with a losartan-hydrochlorothiazide (HCTZ) combination tablet in hypertensive patients who were unable to achieve their target BP. Each tablet contains $50 \mathrm{mg}$ of losartan potassium and $12.5 \mathrm{mg}$ of HCTZ. The efficacy was assessed by changes in BP and the percentage of patients achieving their target BP. Moreover, a stratified analysis was performed at the end of the study to specify in which patient subgroups the losartan-HCTZ tablet was the most effective.

\section{Materials and Methods}

\section{Patients}

The study was a multicenter, open-label, prospective observational study that investigated the efficacy and safety of losartan-HCTZ in hypertensive outpatients. Patients who were 20-80 years old and unable to achieve their target BP (based on JSH 2004 guidelines) with ARB monotherapy at a standard dosage or combination therapy using a standard dosage of ARB with amlodipine $5 \mathrm{mg}$ were recruited. Each ARB was replaced with the losartan-HCTZ tablet after a pre-observation period (1 month). Patients with secondary hypertension, severe heart failure (more than New York Heart Association class III), or renal function impairment (serum creatinine concentration $\geq 2.0 \mathrm{mg} / \mathrm{dL}$ ) were excluded. Fig. 1 shows the study design.
Chronic renal disease and diabetes mellitus were diagnosed by each physician, based on the laboratory analysis measurements. The history of cerebrovascular disease was confirmed by case report forms. The study was performed at 95 centers from September 2007 to December 2009. Case report forms were collected from all patients who received losartan-HCTZ. The study protocol was approved by the ethics committee of each site, and the study complied with the ethical principles of the Declaration of Helsinki. All patients provided their informed consent for their data being used for research purposes before participation in the study.

\section{Treatment}

The patients first received hypotensive therapy with an $\mathrm{ARB}$ at a standard dosage for $\geq 1$ month, either by monotherapy or combination therapy with amlodipine $5 \mathrm{mg}$. Patients unable to achieve their target $\mathrm{BP}$ then received either losartan-HCTZ monotherapy or losartan-HCTZ combination therapy with amlodipine $5 \mathrm{mg}$ once daily for 3 months. No other hypotensive drugs were allowed during this period. Add-on treatment with other antihypertensive agents (amlodipine, diuretic agents, $\beta$-blockers, $\alpha$-blockers, or sympathetic inhibitors) was allowed if the target BP of JSH 2004 $(<140 / 90 \mathrm{mmHg}$ for patients equal or older than age 65 ; $<130 / 85 \mathrm{mmHg}$ for patients younger than age $65 ;<130 / 80$ $\mathrm{mmHg}$ for patients with diabetes mellitus or chronic renal disease; $<140 / 90 \mathrm{mmHg}$ for patients with a history of cerebrovascular disease) was not achieved after 3 months of intensive treatment. The intensive treatment period was 1 year.

\section{Measurement of study variables}

The blood pressure, pulse rate, and dose compliance were recorded at the start of intensive treatment (baseline) and at months 3, 6, 9, and 12. Hematologic tests were done at baseline and at months 3, 6, and 12. The blood measurements included hemoglobin, hematocrit, white blood cell, platelet, low density lipoprotein cholesterol, total cholesterol, triglyceride, high density lipoprotein cholesterol, aspirate aminotransferase, alanine aminotransferase, $\gamma$-glutamyl transpeptidase, lactate dehydrogenase, albumin, total bilirubin, creatine phosphokinase, blood urea nitrogen, uric acid, serum creatinine, natrium, potassium, chlorine, blood glucose and hemoglobin A1c. Blood samples were taken either after fasting or otherwise, provided that it was consistent throughout the study. However, fasting blood samples were mandatory for patients with diabetes mellitus. Blood pressure was measured according to the JSH 2004 guidelines, which advise that outpatient BP should be measured in the seated position after 5 minutes of rest, with caffeine-containing beverages and smoking prohibited within 30 minutes of measurement. BP was measured several times during each session, at 1- to 2-min intervals, and the average of two consecutive stable measurements (i.e. difference $\leq 5 \mathrm{mmHg}$ ) was used. BP measurements were taken from both arms, and the measurement from the arm with the highest BP was used. 


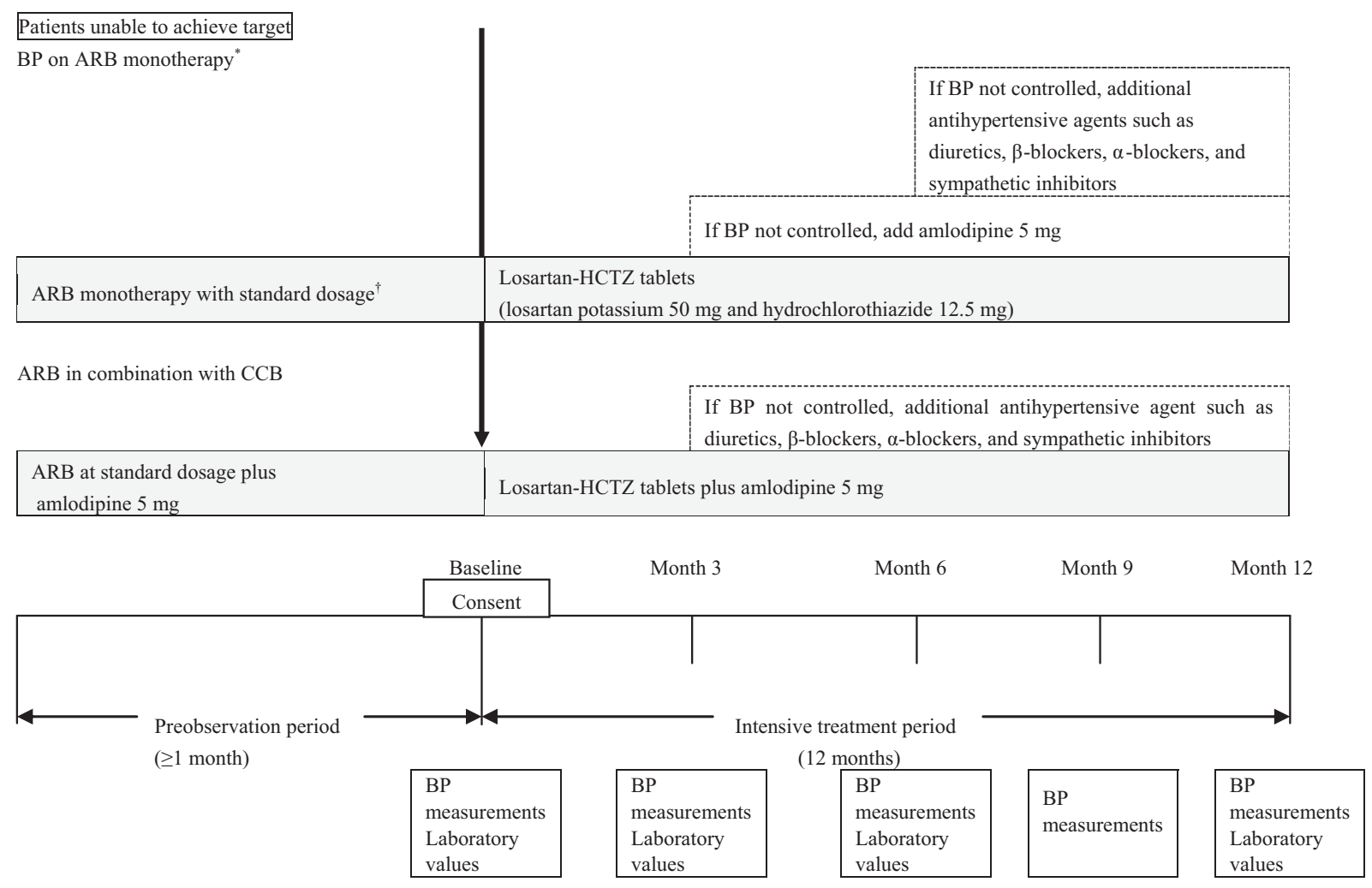

Figure 1. Study design. *Target blood pressure (BP); $<140 / 90 \mathrm{mmHg}$ for patients age 65 or older; $<130 / 85 \mathrm{mmHg}$ for patients younger than age $65 ;<130 / 80 \mathrm{mmHg}$ for patients with diabetes mellitus or chronic renal disease; $<\mathbf{1 4 0 / 9 0} \mathbf{m m H g}$ for patients with a history of cerebrovascular disease. $\dagger$ Angiotensin II receptor antagonist (ARB) at standard dosage: losartan $50 \mathrm{mg}$, valsartan $80 \mathrm{mg}$, candesartan $8 \mathrm{mg}$, olmesartan $20 \mathrm{mg}$, or telmisartan $40 \mathrm{mg}$ daily. CCB: calcium channel blocker, HCTZ: hydrochlorothiazide

\section{Statistical analysis}

The primary endpoint (systolic BP at month 3) was compared with the baseline values using one-sample $t$-test. In addition, a stratified analysis was done on these 3-month changes, stratifying patients by baseline values, gender, alcohol consumption, age, diabetes complications, and body mass index (BMI). Multiple regression analyses were done with the level of effect as the dependent variable to evaluate the effects of various independent variables on changes in systolic and diastolic BP. The independent variables were alcohol consumption, gender, low-density lipoprotein cholesterol concentration, smoking, blood glucose concentration, uric acid concentration, age, and BMI. Other endpoints (systolic BP at month 3, achievement of target BP, and serial changes in BP over 1 year) were analyzed using $t$-test, ANOVA, or the chi-squared test as appropriate. Safety was assessed based on adverse events and clinical laboratory values reported during the study. In addition, summary statistics were collected on safety variables using the means and SD for quantitative data and frequency and proportion for qualitative data.

Results

\section{Patient characteristics}

Case report forms were collected from all 648 patients who received losartan-HCTZ. Data from 614 patients were included in the analysis after 34 patients were excluded because of protocol violations. Summary statistics with means and SD were available for all measurements at each time point. A total of 76 patients (12.4\%) withdrew from or dropped out of the study within 1 year of intensive treatment; the most common reason for withdrawal or dropout was loss to follow-up (30 patients), and 25 patients withdrew or dropped out because of adverse events.

The patient characteristics at baseline are shown in Table 1 . Most of the 614 patients had had hypertension for $<5$ years, and lipid abnormality was the most common associated risk factor. The ARBs most commonly used during the pre-observation period were losartan, valsartan, and candesartan. More patients were in the monotherapy arm (378, $61.6 \%)$ than in the combination therapy arm.

Changes in BP after 3 months of intensive treatment

The mean change in the systolic BP, the primary end- 
Table 1. Baseline Patient Characteristics

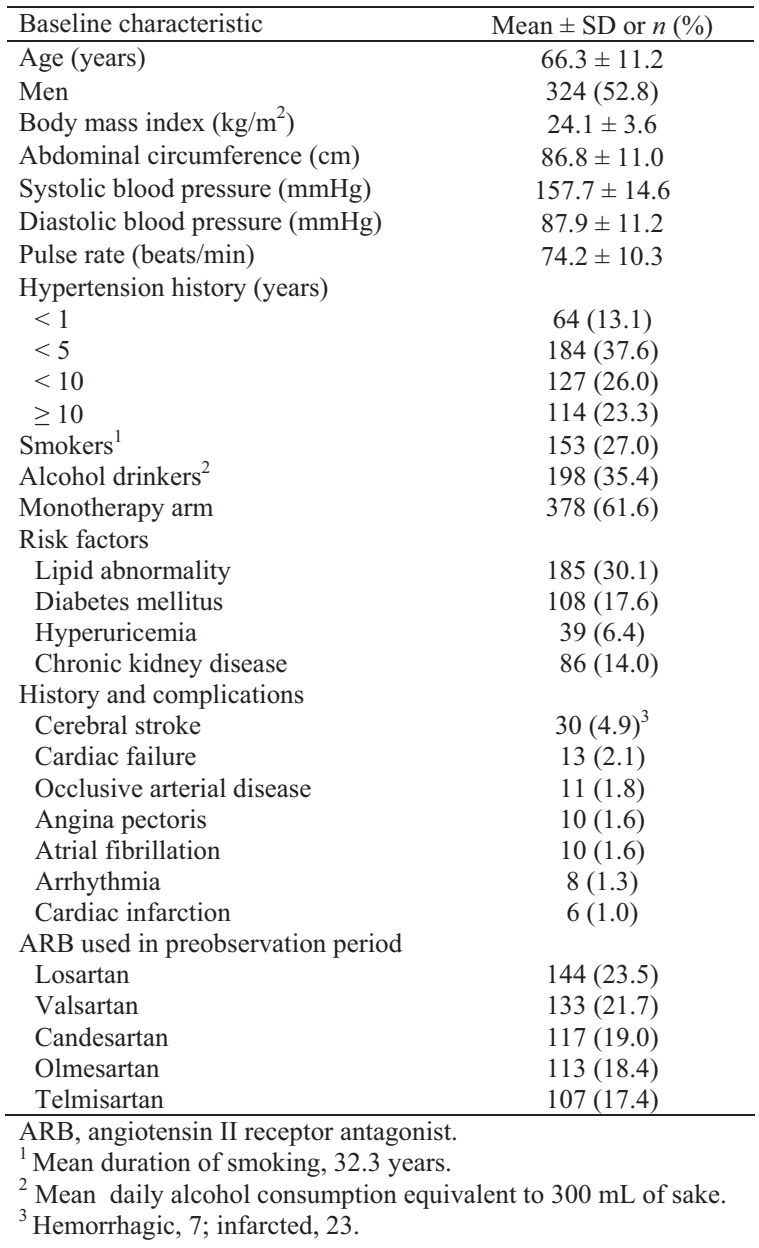

point, in all 614 patients was $-19.7 \mathrm{mmHg}$, a significant decrease from $157.7 \mathrm{mmHg}$ at baseline to $138.0 \mathrm{mmHg}$ at month 3 ( $\mathrm{p}<0.05, t$-test; Fig. $2 \mathrm{~A})$. The mean pulse rate remained almost unchanged (74.2 beats/min at baseline, 73.6 beats/min at month 3 ).

The mean systolic BP in the 354 patients in the losartanHCTZ monotherapy arm at month 3 was $137.4 \mathrm{mmHg}$, a significant decrease of $20.8 \mathrm{mmHg}$ from $158.2 \mathrm{mmHg}$ at baseline ( $<<0.05$, $t$-test; Fig. 2B). Likewise, the mean systolic BP in the 260 patients in the combination therapy arm was $139.4 \mathrm{mmHg}$ at month 3 , a significant decrease of 17.8 $\mathrm{mmHg}$ from $157.2 \mathrm{mmHg}$ at baseline $(\mathrm{p}<0.05, t$-test; Fig. 2C).

The mean change in the diastolic BP in all 614 patients was $-9.7 \mathrm{mmHg}$, a significant decrease from $87.9 \mathrm{mmHg}$ at baseline to $78.2 \mathrm{mmHg}$ at month 3 (p<0.05, $t$-test; Fig. 2A).

The mean diastolic BP in the patients in the monotherapy arm was $78.5 \mathrm{mmHg}$ at month 3 , a significant decrease of $10.4 \mathrm{mmHg}$ from $88.9 \mathrm{mmHg}$ at baseline $(\mathrm{p}<0.05, t$-test; Fig. 2B). Likewise, the mean diastolic BP in the patients in the combination therapy arm was $77.9 \mathrm{mmHg}$ at month 3 , a significant decrease of $8.4 \mathrm{mmHg}$ from $86.3 \mathrm{mmHg}$ at baseline ( $<<0.05$, $t$-test; Fig. $2 \mathrm{C}$ ).

The results showed that $36.2 \%$ of patients had achieved their target BP based on JSH 2004 at month 3 (p<0.05 ver- sus baseline, chi-squared test; data not shown); $39.6 \%$ (243/ $614)$ and $71.7 \%$ (440/614) of the patients had achieved their target systolic and diastolic BP, respectively. This represented a significant increase from $1.8 \%(11 / 614)$ and $36.5 \%$ (224/614), respectively, at baseline $(\mathrm{p}<0.05$, chi-squared test; data not shown).

\section{Patient profiles suited for the losartan-HCTZ combi- nation tablet}

Patients with BP (when seated) which decreased by 20/10 $\mathrm{mmHg}$ at month 3 of the intensive treatment protocol from baseline were categorized as responders to treatment. Patients with systolic BP which decreased by $20 \mathrm{mmHg}$ at month 3 of intensive treatment from baseline were categorized as systolic BP responders. Likewise, patients with diastolic BP which decreased by $10 \mathrm{mmHg}$ at month 3 and/or from baseline were categorized as diastolic BP responders. At month 3,70.8\% of patients (435/614) were systolic BP responders, $88.9 \%(546 / 614)$ were diastolic BP responders, and $61.7 \%(379 / 614)$ were systolic and diastolic BP responders (data not shown).

Systolic BP responders and non-responders were compared for each relevant patient characteristic. Age, BMI, smoking, concurrent diseases, and ARB treatment during the pre-observation period showed no significant difference between the groups. However, significant differences were found for gender and alcohol consumption; the percentages of responders and non-responders were 50.1\% (218/435) and $59.2 \%(106 / 179)$ for gender (men) and 32.8\% (131/399) and $41.9 \%$ (67/160) for alcohol consumption (both $\mathrm{p}<0.05$, chi-squared test, data not shown).

Stratified analyses of changes in systolic BP from baseline to month 3 were done according to the following stratifications: baseline systolic BP $(\geq 160 \mathrm{mmHg}$ or $<160$ $\mathrm{mmHg}$ ), gender, alcohol consumption, smoking, age ( $\geq 65$ years or $<65$ years), diabetes, hyperlipidemia, hyperuricemia and BMI $\left(\geq 25 \mathrm{~kg} / \mathrm{m}^{2}\right.$ or $\left.<25 \mathrm{~kg} / \mathrm{m}^{2}\right)$. Therefore, patients with high baseline systolic BP, women, patients who did not drink alcohol, and elderly patients (aged $\geq 65$ years) showed a significantly greater hypotensive response $(\mathrm{p}<0.001$, unpaired $t$-test, Table 2A). A multiple regression analysis was done using the influences on changes in systolic BP as dependent variables. Only alcohol consumption significantly affected changes in the systolic BP ( $\mathrm{p}=0.045$; Table $3 \mathrm{~A})$.

Furthermore, stratified analyses of changes in diastolic BP from baseline at month 3 were performed as in systolic BP. Table 2B showed that the hypotensive effect of the losartan/ HCTZ tablet was significantly greater in patients with high baseline diastolic BP $(\geq 100 \mathrm{mmHg})$, patients who did not drink alcohol, and younger patients (aged $<65$ years). Women tended to have a lower BP after 3 months of treatment, although the difference was not significant. A multiple regression analysis revealed that alcohol consumption and gender significantly affected the changes in diastolic BP ( $\mathrm{p}=$ 0.001, 0.043, respectively, Table 3B). 

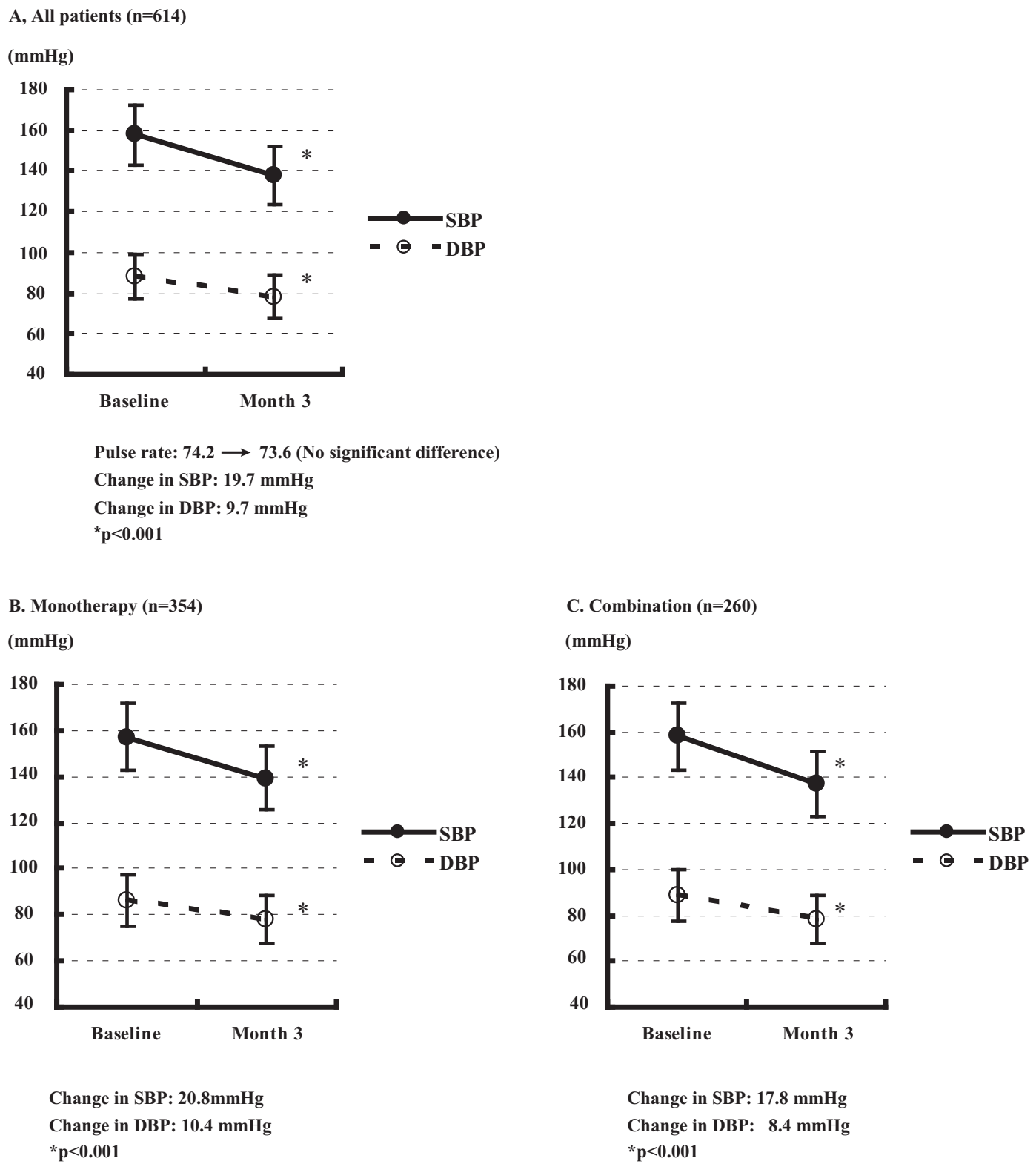

Change in SBP: $20.8 \mathrm{mmHg}$

Change in DBP: $10.4 \mathrm{mmHg}$ *p $<0.001$

${ }^{*} \mathbf{p}<\mathbf{0 . 0 0 1}$

Figure 2. Changes in the blood pressure (means \pm SD) at month 3 of intensive treatment with losartan-hydrochlorothiazide: a, all patients $(n=614)$; b, monotherapy arm alone $(n=354)$; and c, combination therapy arm alone $(n=260)$. $* p<0.05$ ( $t$-test $)$.

\section{Efficacy and safety of losartan-HCZ based therapy during 1 year of treatment}

Both systolic and diastolic BP decreased significantly by month 3, and the hypotensive effect persisted for 1 year $(\mathrm{p}<$ 0.001, ANOVA; Fig. 3). An adequate hypotensive effect was found soon after intensive treatment was begun, and BP was well controlled over 1 year, although add-on drugs were often provided after month 3 . The pulse rate remained unchanged from baseline through 1 year.

The laboratory measurements that changed significantly at month 3 were hematocrit, concentrations of total cholesterol, lactate dehydrogenase, blood urea nitrogen, serum creatinine, sodium, potassium, and chloride. Overall, these changes were within reference ranges and considered not clinically relevant. Measurements sensitive to diuretic agents, such as blood glucose concentration, hemoglobin A1c value, and uric acid concentration, showed no significant changes. The fasting blood glucose concentration, hemoglobin A1c value, and lipid concentrations other than total cholesterol (low-density lipoprotein cholesterol, highdensity lipoprotein cholesterol, and triglyceride concentration) also showed no significant changes (paired $t$-test).

Only $5.2 \%$ of the patients (32/614) experienced a total of 35 adverse events over the entire observation period. The adverse events experienced by at least two patients included; dizziness in eight patients (1.3\%), and hypokalemia, hypotension, rash, diarrhea, impaired liver function, impaired renal function, and cerebral infarction, each of which occurred in two patients $(0.3 \%)$. These events were mostly mild, none were severe, and they resolved during the study. Hypokalemia, which is commonly associated with diuretic 
Intern Med 51: 1167-1175, 2012 DOI: 10.2169/internalmedicine.51.6297

Table 2. Stratified Analysis of Changes in Blood Pressure after 3 Months of Intensive Treatment

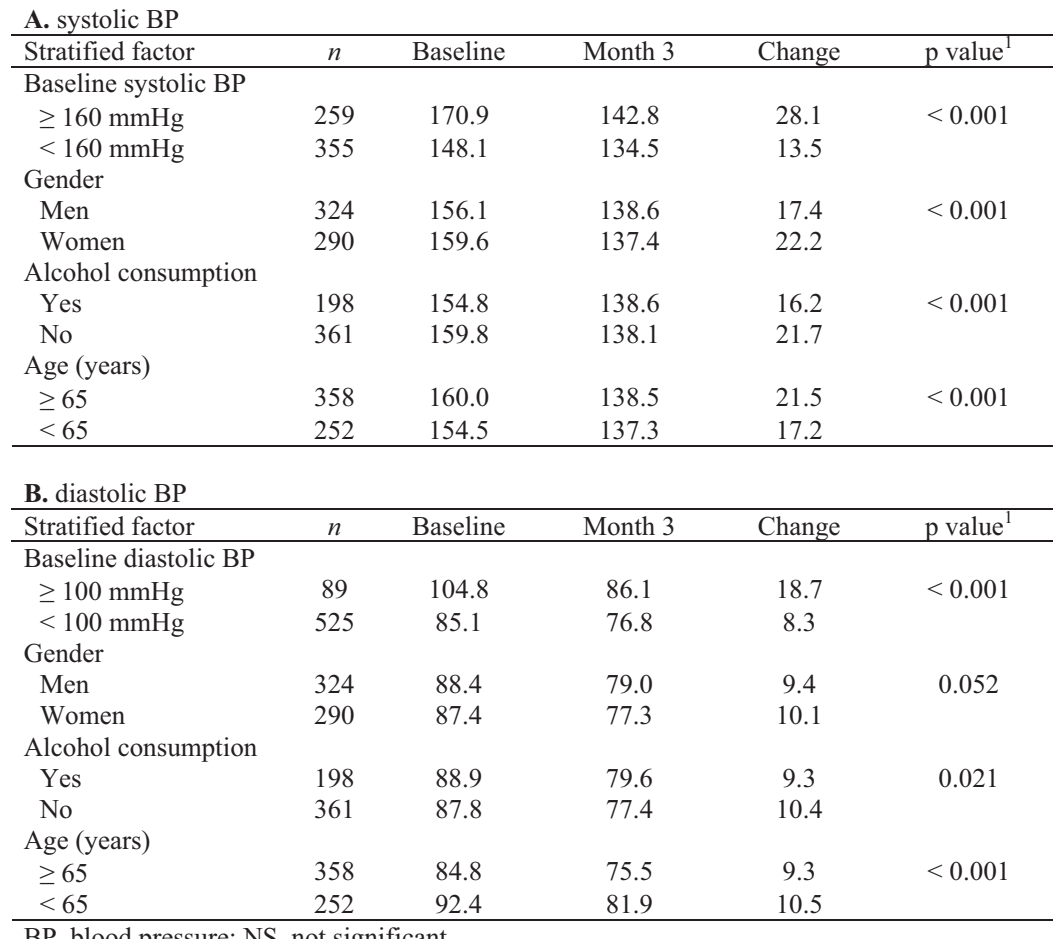

$\mathrm{BP}$, blood pressure; NS, not significant.

${ }^{1}$ Unpaired $t$-test.

Table 3. Effect on Changes in Systolic Blood Pressure (Multiple Regression)

\begin{tabular}{|c|c|c|c|c|c|}
\hline $\begin{array}{l}\text { A. systuence } \\
\text { rank }\end{array}$ & $\begin{array}{c}\text { Independent } \\
\text { variable }\end{array}$ & $\begin{array}{c}\text { Partial } \\
\text { regression } \\
\text { coefficient }\end{array}$ & $\begin{array}{c}\text { Standardized } \\
\text { partial } \\
\text { regression } \\
\text { coefficient }\end{array}$ & $\mathrm{t}$ value & $\mathrm{p}$ value \\
\hline & Constant & 31.784 & & 1.464 & 0.148 \\
\hline 1 & $\begin{array}{c}\text { Alcohol } \\
\text { consumption }\end{array}$ & -9.304 & -0.293 & -1.995 & 0.045 \\
\hline 2 & Gender & 6.440 & 0.211 & 1.487 & 0.142 \\
\hline 3 & $\begin{array}{c}\text { LDL-C } \\
\text { concentration }\end{array}$ & -0.058 & -0.103 & -0.829 & 0.410 \\
\hline 4 & Smoking & 3.302 & 0.096 & 0.713 & 0.479 \\
\hline 5 & $\begin{array}{l}\text { Blood glucose } \\
\text { concentration }\end{array}$ & -0.032 & -0.086 & -0.724 & 0.471 \\
\hline 6 & $\begin{array}{l}\text { Uric acid } \\
\text { concentration }\end{array}$ & -0.271 & -0.026 & -0.203 & 0.840 \\
\hline 7 & Age & 0.015 & 0.010 & 0.083 & 0.934 \\
\hline 8 & Body mass index & -0.048 & -0.010 & -0.080 & 0.937 \\
\hline \multicolumn{6}{|c|}{ B. diastolic BP } \\
\hline $\begin{array}{l}\text { Influence } \\
\text { rank }\end{array}$ & $\begin{array}{c}\text { Independent } \\
\text { variable }\end{array}$ & $\begin{array}{c}\text { Partial } \\
\text { regression } \\
\text { coefficient }\end{array}$ & $\begin{array}{c}\text { Standardized } \\
\text { partial } \\
\text { regression } \\
\text { coefficient }\end{array}$ & t value & $\mathrm{p}$ value \\
\hline & Constant & 35.875 & & 2.380 & 0.020 \\
\hline 1 & $\begin{array}{c}\text { Alcohol } \\
\text { consumption }\end{array}$ & -5.509 & -0.480 & -3.360 & 0.001 \\
\hline 2 & Gender & 3.153 & 0.286 & 2.070 & 0.043 \\
\hline 3 & Age & -0.211 & -0.205 & -1.690 & 0.096 \\
\hline 4 & $\begin{array}{c}\text { LDL-C } \\
\text { concentration }\end{array}$ & -0.055 & -0.135 & -1.120 & 0.269 \\
\hline 5 & $\begin{array}{l}\text { Blood glucose } \\
\text { concentration }\end{array}$ & -0.032 & -0.116 & -1.010 & 0.316 \\
\hline 6 & $\begin{array}{c}\text { Uric acid } \\
\text { concentration }\end{array}$ & -0.940 & -0.126 & -1.000 & 0.321 \\
\hline 7 & Body mass index & 0.086 & 0.025 & 0.200 & 0.839 \\
\hline 8 & Smoking & 0.140 & 0.011 & 0.090 & 0.932 \\
\hline
\end{tabular}

BP, blood pressure; LDL-C, low-density lipoprotein cholesterol. 


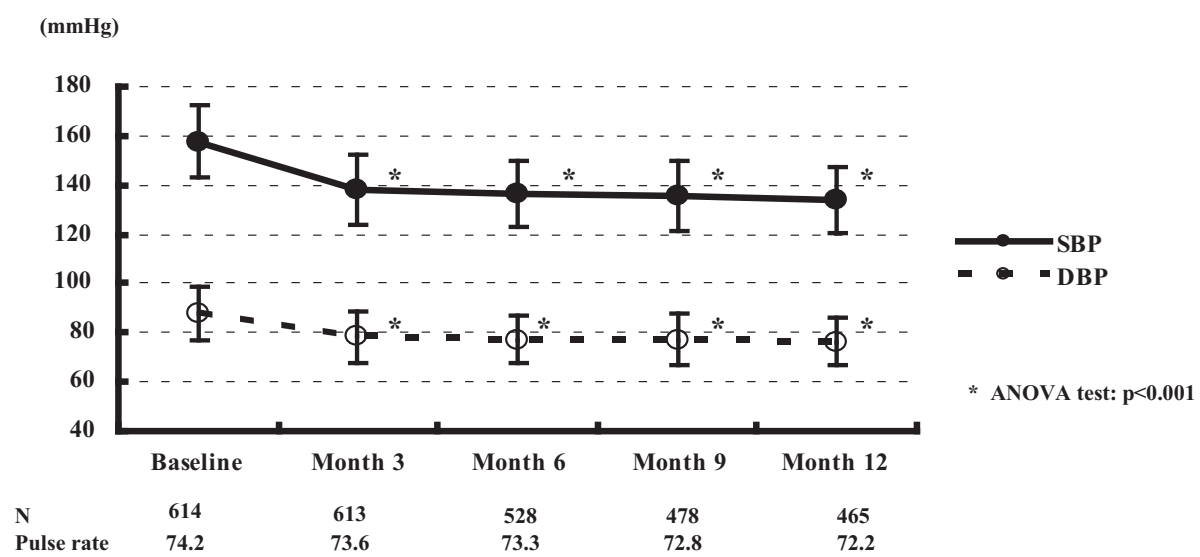

Figure 3. Changes in the blood pressure (means \pm SD) over 1 year of intensive treatment with losartan-hydrochlorothiazide. *p<0.05 (ANOVA).

agents, occurred in only three patients, and all cases were mild. All adverse events that occurred during the study have been previously reported.

\section{Discussion}

The present study confirmed the efficacy of the losartanHCTZ combination tablet, and no excessive hypotension was noted with the combination of two active ingredients. The results in the present study proved the safety of the long-term use of losartan-HCTZ. Intensive treatment with losartan-HCTZ for patients with hypertension uncontrolled by ARB therapy is particularly effective in patients with high baseline BP and in women. On the other hand, losartan-HCTZ is less effective in patients who drink alcohol, thus suggesting the importance of lifestyle intervention as well as drug therapy to treat hypertension.

Hypertension is a crucial risk factor for potentially fatal complications such as cerebrovascular and cardiovascular diseases. The meta-analysis indicated that BP should be appropriately controlled in patients with hypertension to improve their prognosis (3). Unfortunately, only 50\% of patients treated with a single antihypertensive agent achieve their target BP (4-8). Therefore, the guidelines for hypertension treatment recommend combination therapy with antihypertensive agents, which is more effective in reducing BP than an increased dosage of a single agent in patients whose BP is uncontrolled by monotherapy $(9,10)$. The combination of a RAS inhibitor and a diuretic agent is recommended by the Japanese, US, and European guidelines. Although substantial reductions in BP from baseline were achieved by losartan/HCTZ therapy, only $36.2 \%$ of patients achieved the target $\mathrm{BP}$ of $<140 / 90 \mathrm{mmHg}$ by month 3 of intensive treatment, thus indicating the difficulty in achieving a strict control of BP. Actually, more than half of the patients required combination therapy with three or more drugs, including ARBs, diuretic agents, and CCBs during the observation period.

The stratified analyses of changes in systolic BP from baseline to month 3 showed significantly greater hypoten- sive effects in patients with high baseline systolic BP, women, patients who do not drink alcohol, and patients $\geq 65$ years. It is plausible that general pharmacological effects of antihypertensive drugs contribute to the association between higher baseline BP and greater hypotensive effect. Elderly and female patients have clinical features of salt-sensitive hypertension which might contribute to greater hypotensive effect of losartan-HCTZ. Elderly patients usually have low renin and low aldosterone concentration and are generally considered to have volume retention associated with renal dysfunction (11). Therefore, many elderly patients tend to be salt-sensitive and show a good response to diuretic agents. In addition, the combination of multiple drugs into a single tablet could improve adherence to treatment particularly in elderly patients. The present study shows losartanHCTZ tablets to be a combination agent with significant hypotensive effects in elderly patients without severe complications and therefore they are particularly useful for treating hypertension in this group of patients.

Responses to RAS inhibitors differ between genders; women tend to have lower renin activity, and men tend to respond better to RAS inhibitors (12). One characteristic of hypertension in women is that the prevalence of hypertension greatly increases after menopause $(9,10)$. An imbalance of estrogens and progesterone leads menopausal women to fluid retention, which might be associated with a salt-sensitive hypertension in women (13). The diuretic effect of losartan-HCTZ activates the RAS as a result of volume loss, leading to the synergistic hypotensive effect of ARB, losartan. The present study found a significantly strong hypotensive effect in women, thus suggesting that losartan-HCTZ is effective in women with hypertension and a low renin activity.

It is interesting to note that younger patients achieved a greater decrease in their diastolic BP, while older patients achieved a larger decrease in their systolic BP. One of the characteristics of hypertension in elderly patients is isolated systolic hypertension caused by arterial stiffening. Actually, the baseline diastolic BP was lower in elderly patients, while the baseline systolic BP was higher (Table 2). Al- 
though speculative, it is plausible that more severe arterial stiffening in elderly patients attenuated the vasodilating effect of the losartan/HCTZ tablet.

A multiple regression analysis was performed on various factors influencing changes in BP. Only alcohol consumption has a significant negative effect on changes in both systolic and diastolic BP. A long period of alcohol intake increases BP (14). In addition, previous reports have indicated that the less alcohol a patient drinks, the lower his or her BP tends to be (15). As a result, reducing alcohol consumption by $80 \%$ has been demonstrated to rapidly reduce BP (16). Although the present study did not analyze the diet and lifestyle of the study population in detail, it is likely that people who drink more alcohol generally have a more disordered diet and lifestyle than those who do not drink alcohol. The attenuated hypotensive effect in drinkers might therefore be associated with these problems.

Two drug combinations, ARB-diuretic agent and ARB$\mathrm{CCB}$, are used in clinical practice in Japan. Hypertension specialists suggest that patients with hypertension that is uncontrolled by RAS inhibitors should therefore receive either combination therapy with diuretic agents if the disease is associated with excessive fluid retention, or combination therapy with CCB if it is associated with arteriosclerosis. The results of future comparison studies to elucidate the optimal usage of such losartan-HCTZ combination tablets and the ARB-CCB are therefore highly anticipated.

\section{Author's disclosure of potential Conflicts of Interest (COI).}

Murohara T: Honoraria, MSD and Pfizer. Koh N: Honoraria, MSD.

\section{Acknowledgement}

We express our deep gratitude for the support of all physicians who kindly participated in this study bellow. Without their support, the collection of real world data would have been impossible. This study was supported by Japan Heart Foundation.

Members of the ARCH Study Group: Kenji Yamada, Masayuki Nakamura, Hideo Takeda, Hiroshi Anazawa, Kengo Kanemaki, Sumihiko Hayashi, Hideo Tanaka, Fumihiro Imai, Kenji Yasui, Yukio Kawata, Tetsuya Kubota, Toshio Aoki, Hisayoshi Hirota, Keisuke Takagi, Yoshitaka Miura, Fumiro Isaji, Makoto Kutsuna, Hirohiko Nomura, Tatsuhiko Narita, Koichi Nakada, Katsushi Nishizawa, Hayato Sugiura, Kazuhiko Okamura, Kenichi Hayakawa, Yukio Watanabe, Hideo Nomura, Bunyu Ogasawara, Masayuki Suzuki, Shigeki Horita, Yoshiaki Ishiguro, Kiyoshi Iimi, Masayoshi Adachi, Akisada Ando, Sawao Ishikawa, Naoki Kawai, Masaharu Hosoi, Jiro Riuge, Kazuya Domitsu, Sadao Oguchi, Harumichi Kato, Mitsumoto Hoshiai, Tadashi Ito, Junko Ito, Tatsuya Kato, Toshihiko Kamiya, Kenichi Ohara, Hidetaka Shishido, Yukari Tomomatsu, Hideo Wakayama, Hidefumi Iwatsuki, Ryo Kumagai, Hiroshi Inui, Kakuro Okumura, Norishige Ozeki, Kiwamu Tokoro, Hidehiko Jose, Kazuko Shimono, Hiroshi Kurokawa, Norio Yamane, Yoshisuke Takada, Hiroshi Kakehi, Goro Narita, Hideo Imai, Hirofumi Kanda, Isao Koishikawa, Haruo Kaneko, Kuniyoshi Kojima, Kenji Imai,
Masuo Kawamura, Mikihiro Nakayama, Masataka Tajima, Minoru Tanaka, Kazuo Otake, Koh Matsuo, Fumihiro Mitsuguchi, Toshifumi Sakakibara, Masaichi Hasegawa, Eisuke Takeshima, Kazuhito Ichikawa, Tomiji Kasio, Atsushi Kinoshita, Yutaka Kaneko, Kazuhide Takami, Akira Nakai, Hiroshi Sato, Akihisa Uemura, Hideaki Kuroki, Yasunaga Noda, Toshihiro Ohama, Fumitada Kamiya, and Fuminori Ito.

\section{References}

1. Intersalt Cooperative Research Group. Intersalt: an international study of electrolyte excretion and blood pressure. Results for 24 hour urinary sodium and potassium excretion. BMJ 297: 319-328, 1988.

2. Ohta Y, Tsuchihashi T, Onaka U, Eto K, Ueno M. Usefulness of the $\alpha_{1}$-blocker doxazosin as a third-line antihypertensive drug. Hypertens Res 30: 301-306, 2007.

3. Staessen JA, Wang JG, Thijs L. Cardiovascular protection and blood pressure reduction: a meta-analysis. Lancet 358: 1305-1315, 2001.

4. Manolis AJ, Grossman E, Jelakovic B, et al. Losartan Trial Investigators. Effects of losartan and candesartan monotherapy and losartan/hydrochlorothiazide combination therapy in patients with mild to moderate hypertension. Clin Ther 22: 1186-1203, 2000.

5. Chan JCN, Critchley JAJH, Lappe JT, et al. Randomised, doubleblind, parallel study of the anti-hypertensive efficacy and safety of losartan potassium compared with felodipine ER in elderly patients with mild to moderate hypertension. J Hum Hypertens 9: 765-771, 1995.

6. Dahlöf B, Keller Se, Makris L, Goldberg AI, Sweet CS, Lim NY. Efficacy and tolerability of losartan potassium and atenolol in patients with mild to moderate essential hypertension. Am J Hypertens 8: 578-583, 1995.

7. Tikkanen I, Omvik P, Jensen HA. Comparison of the angiotensin II antagonist losartan with the angiotensin converting enzyme inhibitor enalapril in patients with essential hypertension. J Hypertens 13: 1343-1351, 1995.

8. Mallion JM, Bradstreet DC, Makris L, et al. Antihypertensive efficacy and tolerability of once daily losartan potassium compared with captopril in patients with mild to moderate essential hypertension. J Hypertens Suppl 13: S35-S41, 1995.

9. Dahlöf B, Devereux RB, Kjeldsen SE, et al.; LIFE Study Group. Cardiovascular morbidity and mortality in the Losartan Intervention For Endpoint reduction in hypertension study (LIFE): a randomised trial against atenolol. Lancet 359: 995-1003, 2002.

10. Dahlöf B, Sever PS, Poulter NR, et al.; ASCOT Investigators. Prevention of cardiovascular events with an antihypertensive regimen of amlodipine adding perindopril as required versus atenolol adding bendroflumethiazide as required, in the Anglo-Scandinavian Cardiac Outcomes Trial-Blood Pressure Lowering Arm (ASCOTBPLA): a multicentre randomised controlled trial. Lancet 366: 895-906, 2005.

11. Luft FC, Weinberger MH, Fineberg NS, Miller JZ, Grim CE. Effects of age on renal sodium homeostasis and its relevance to sodium sensitivity. Am J Med 82: 9-15, 1987.

12. Staessen JA, Celis H, Fagard R. The epidemiology of the association between hypertension and menopause. J Hum Hypertens 12: 587-592, 1998.

13. Luoto R, Sharrett AR, Schreiner P, Sorlie PD, Arnett D, Ephross S. Blood pressure and menopausal transition: the Atherosclerosis Risk in Communities study (1987-95). J Hypertens 18: 27-33, 2000.

14. Nakamura K, Okamura T, Hayakawa T, et al.; NIPPON DATA 90 Research Group. The proportion of individuals with alcoholinduced hypertension among total hypertensives in a general Japa- 
Intern Med 51: 1167-1175, 2012 DOI: 10.2169/internalmedicine.51.6297

nese population: NIPPON DATA 90. Hypertens Res 30: 663-668, 2007.

15. Xin X, He J, Frontini MG, Ogden LG, Motsamai OI, Whelton PK. Effects of alcohol reduction on blood pressure: a metaanalysis of randomized controlled trials. Hypertension 38: 11121117,2001
16. Puddey IB, Beilin LJ, Vandongen R. Regular alcohol use raises blood pressure in treated hypertensive subjects. A randomised controlled trial. Lancet 1: 647-651, 1987.

(C) 2012 The Japanese Society of Internal Medicine http://www.naika.or.jp/imindex.html 